\title{
A Sleep Apnea Detection System Based on a One-Dimensional Deep Convolution Neural Network Model Using Single-Lead Electrocardiogram
}

\author{
Hung-Yu Chang ${ }^{1,2}$, Cheng-Yu Yeh ${ }^{3}$, Chung-Te Lee ${ }^{3}$ and Chun-Cheng Lin ${ }^{3, *}$ \\ 1 Heart Center, Cheng Hsin General Hospital, Taipei 112, Taiwan; amadeus0814@yahoo.com.tw \\ 2 Faculty of Medicine, School of Medicine, National Yang Ming University, Taipei 112, Taiwan \\ 3 Department of Electrical Engineering, National Chin-Yi University of Technology, Taichung 41170, Taiwan; \\ cy.yeh@ncut.edu.tw (C.-Y.Y.); chunghengnice1@gmail.com (C.-T.L.) \\ * Correspondence: cclin@ncut.edu.tw; Tel.: +886-4-2392-4505 (ext. 7238)
}

Received: 24 June 2020; Accepted: 24 July 2020; Published: 26 July 2020

check for updates

\begin{abstract}
Many works in recent years have been focused on developing a portable and less expensive system for diagnosing patients with obstructive sleep apnea (OSA), instead of using the inconvenient and expensive polysomnography (PSG). This study proposes a sleep apnea detection system based on a one-dimensional (1D) deep convolutional neural network (CNN) model using the single-lead 1D electrocardiogram (ECG) signals. The proposed CNN model consists of 10 identical CNN-based feature extraction layers, a flattened layer, 4 identical classification layers mainly composed of fully connected networks, and a softmax classification layer. Thirty-five released and thirty-five withheld ECG recordings from the MIT PhysioNet Apnea-ECG Database were applied to train the proposed $\mathrm{CNN}$ model and validate its accuracy for the detection of the apnea events. The results show that the proposed model achieves $87.9 \%$ accuracy, $92.0 \%$ specificity, and $81.1 \%$ sensitivity for per-minute apnea detection, and $97.1 \%$ accuracy, $100 \%$ specificity, and $95.7 \%$ sensitivity for per-recording classification. The proposed model improves the accuracy of sleep apnea detection in comparison with several feature-engineering-based and feature-learning-based approaches.
\end{abstract}

Keywords: obstructive sleep apnea; single-lead electrocardiogram; deep learning; convolutional neural network

\section{Introduction}

Obstructive sleep apnea (OSA) is a common sleep disorder that can cause shortness of breath or cessation of breathing, and is characterized by repeated pharyngeal collapse causing partial or complete obstruction of the upper airway, affecting ventilation during sleep [1]. It results in insufficient air entering the lungs and decreased blood oxygen concentration. Because of the lack of oxygen in the brain, patients are more likely to wake up, and so their sleep is frequently interrupted. OSA is highly prevalent in patients with cardiovascular disease, and is associated with the incidence and morbidity of hypertension, coronary heart disease, arrhythmia, heart failure, and stroke [2]. Polysomnography (PSG) is considered to be the gold standard for diagnosing OSA. It has to be done overnight in a sleep laboratory or sleep center, and requires several sensors to record multiple sleep physiological signals, including electroencephalography (EEG), electrooculogram (EOG), electrocardiogram (ECG), electromyogram (EMG), $\mathrm{SaO} 2$ saturation, thoracic abdominal effort, nasal-oral airflow, blood pressure, heart rate, and leg movement [3,4]. PSG measures the apnea-hypopnea index (AHI), which is defined as the sum of apneas and hypopneas per hour of sleep and has been widely used for diagnosing patients with OSA. According to the AHI, the severity of OSA can be classified as none $(\mathrm{AHI}<5)$, mild $(5 \leq \mathrm{AHI}<15)$, moderate $(15 \leq \mathrm{AHI}<30)$, or severe (AHI $\geq 30)$ [5]. 
However, as PSG is an inconvenient, time-consuming, and expensive examination system, many studies in recent years have focused on the development of a portable and less expensive OSA diagnostic system using fewer physiological signals including blood oxygen concentration, ECG, chest and abdominal respiratory signals, breathing sounds, and the combined signals [6]. Evidence supports that single-lead ECG-based detection methods provide the highest global classification by studied population ratio among the algorithms based on a single-source sensor [6]. Analysis of ECG waveforms [7-9] and ECG-derived heart rates [10-14] are commonly used to detect sleep disordered breathing, including apnea, and hypopnea events.

Most of the previous works focused on feature engineering which requires a specific feature extraction method to extract ECG features from the ECG waveforms, RR intervals, heart rate variability (HRV), and ECG-derived respiration (EDR) signals. For example, several studies introduced the wavelet decomposition to extract the features from ECG waveforms [7-9]. Nguyen et al. [10] calculated the recurrence quantification analysis statistics of HRV signals to capture the dynamic changes of the cardiorespiratory system during OSA. Atri and Mohebbi [11] extracted a set of features from the bispectrum of HRV and EDR to acquire their nonlinear and non-Gaussian properties. Sharma and Sharma [12] extracted 16 features using the Hermite approximation of QRS waveforms including the Hermite expansion coefficients and the approximation error energy, and two features from RR intervals including the mean value and standard deviation of RR intervals. Song et al. [13] proposed a feature extraction method based on a hidden Markov model, and a feature selection approach using leave-one-out cross-validation to remove trivial features. Varon et al. [14] extracted two features derived from the changes in the morphology of QRS complexes and from heart rate and EDR using the orthogonal subspace projections. These two features were further combined with two features from the parameters of HRV including the standard deviation and the serial correlation coefficient. The classification techniques used by the previous studies include neural networks [7,10,12], K-Nearest Neighbor (KNN) [12,13], support vector machines (SVM) [8,10,12-14], random under sampling boosting [9], least-square SVM [11,12,14], the hidden Markov model [13], and linear discriminant analysis (LDA) [13,14].

Recently, several studies have proposed neural networks to automatically learn features. Singh and Majumder [15] proposed a pre-trained two-dimensional (2D) AlexNet model based on a convolutional neural network $(\mathrm{CNN})$ to extract the features from 2D time-frequency images of ECG signals, and a decision fusion method consisting of the KNN, SVM, LDA, and Ensemble classifiers to improve the sensitivity for detecting apnea events. Wang et al. [16] proposed a modified LeNet-5 CNN model to extract features from 1D ECG signals and RR intervals, and to classify the normal and apnea events. Li et al. [17] developed a deep neural network to learn features from RR intervals, which belongs to unsupervised learning that only requires unlabeled ECG signals, and designed two types of classifiers including SVM and artificial neural network (ANN) to detect the apnea events.

All of the above-mentioned techniques have demonstrated their superiority in the detection of apnea events. However, this study aims to further simplify the complexity of the signal preprocessing for the design of the CNN model. This study therefore proposes a sleep detection system based on a 1D deep CNN model and the use of single-lead 1D ECG signals. Only 1D ECG signals served as the input. The detection of QRS complexes and the analysis of ECG-derived heart rates are not required in this study. The signal preprocessing only includes Butterworth bandpass filtering and z-score normalization. Because the proposed CNN model has the same dimension of $1 \mathrm{D}$ with the input ECG signals, the signal preprocessing does not need additional signal transformation.

The rest of this paper is organized as follows. Section 2 describes the ECG recordings obtained from the MIT PhysioNet Apnea-ECG database, and presents the proposed apnea detection system based on a 1D deep CNN model. Results are given in Section 3. A discussion of the study findings is provided in Section 4. Finally, Section 5 concludes this study. 


\section{Materials and Methods}

\subsection{Materials}

The MIT PhysioNet Apnea-ECG database [18,19] was recruited in this study. This database consists of a released dataset of 35 recordings and a withheld dataset of 35 recordings, digitized with at sampling rate of $100 \mathrm{~Hz}$ and with 12-bit resolution. The recording length varies from 401 to $587 \mathrm{~min}$. Each recording contains a single-lead ECG signal and a set of annotations. Each 1-min ECG signal is annotated as label $\mathrm{N}$ or $\mathrm{A}$, where $\mathrm{N}$ and $\mathrm{A}$ denote the normal and apnea events, respectively. All apnea events are obstructive or mixed. The events of pure central apnea and Cheyne-Stoke respiration are not included in the database. If a 1-min ECG signal contains hypopneas which have intermittent decreases in respiratory flow of less than $50 \%$ and decreases in oxygen saturation of at least $4 \%$, and is accompanied by compensating hyperventilation, it is also annotated as apnea. These ECG recordings are divided into Class A (Apnea), Class B (Borderline), and Class C (Control). Recordings in classes $A$ and $B$ include at least one hour with an apnea index of 10 or more, and of 5 or more, respectively. Moreover, recordings in classes A, B, and C contain apnea or hypopnea of at least 100 min, between 5 and $99 \mathrm{~min}$, and fewer than 5 min during the recording, respectively. The released and withheld datasets each contain 20 recordings of Class A, 5 recordings of Class B, and 10 recordings of Class C.

The released dataset was used for training the proposed 1D deep CNN model, and the withheld dataset was used to validate the performance of the proposed model. A total of 34,213 1-min ECG signals were extracted from the released and withheld datasets in this study. The released dataset has 16,979 $\mathrm{min}$, of which 10,322 and $6657 \mathrm{~min}$ are annotated as normal and apnea events, respectively. The withheld dataset has 17,234 min, of which 10,717 and $6517 \mathrm{~min}$ are annotated as normal and apnea events, respectively.

\subsection{The Proposed Apnea Detection System Based on a 1D Deep CNN Model}

Figure 1 shows the block diagram of the proposed sleep apnea detection system based on a 1D deep CNN model. The input signal is a 1-min ECG signal with a length of 6000 samples in released and withheld sets. The signal preprocessing includes band-pass filtering and standardization. Each 1-min ECG signal was filtered through a fourth-order Butterworth bandpass filter with a $0.5 \mathrm{~Hz}$ to $15 \mathrm{~Hz}$ passband to reduce baseline drift and high-frequency interference. The Butterworth bandpass filter at $100 \mathrm{~Hz}$ sampling rate is implemented using the butter function from the Matlab signal processing toolbox [20]. Its difference equation is given as

$$
\begin{aligned}
\mathrm{y}(\mathrm{n})= & 0.1242 \mathrm{x}(\mathrm{n})-0.2483 \mathrm{x}(\mathrm{n}-2)+0.1242 \mathrm{x}(\mathrm{n}-4)+ \\
& 2.7422 \mathrm{y}(\mathrm{n}-1)-2.7907 \mathrm{y}(\mathrm{n}-2)+1.3311 \times \mathrm{y}(\mathrm{n}-3)-0.2831 \mathrm{y}(\mathrm{n}-4)
\end{aligned}
$$

The z-score function was used for standardizing the filtered ECG signals defined as follows:

$$
\mathrm{z}=\frac{\mathrm{x}-\mu}{\sigma}
$$

where $\mathrm{x}$ is the input signal, and $\mu$ and $\sigma$ are the mean and standard deviation of $\mathrm{x}$, respectively. The $\mathrm{z}$ score shows how many standard deviations the input signal is from the mean. Figure 2 shows an illustration of the 1-min ECG signal (top) and the signals after bandpass filtering (middle) and z-score normalization (bottom). It is shown that most of the baseline drift can be removed after the bandpass filtering. The amplitudes of the filtered ECG signal ranging from $-435 \mu \mathrm{V}$ to $412 \mu \mathrm{V}$ were reduced to amplitudes ranging from $-4.8 \mu \mathrm{V}$ to $4.6 \mu \mathrm{V}$ after z-score normalization. 


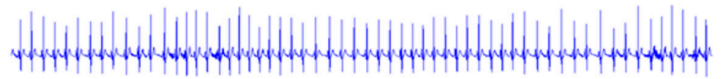

1-minute ECG in released and withheld datasets

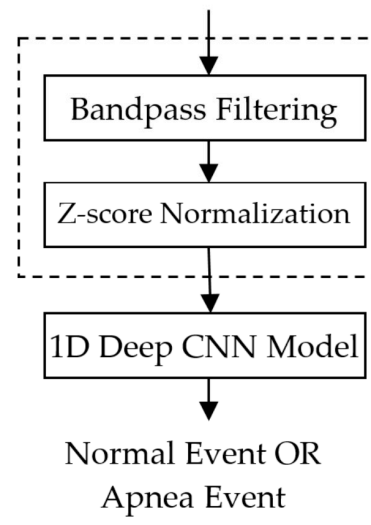

Signal Preprocessing

Figure 1. Block diagram of the proposed sleep apnea detection system based on a 1D deep convolutional neural network (CNN) model.

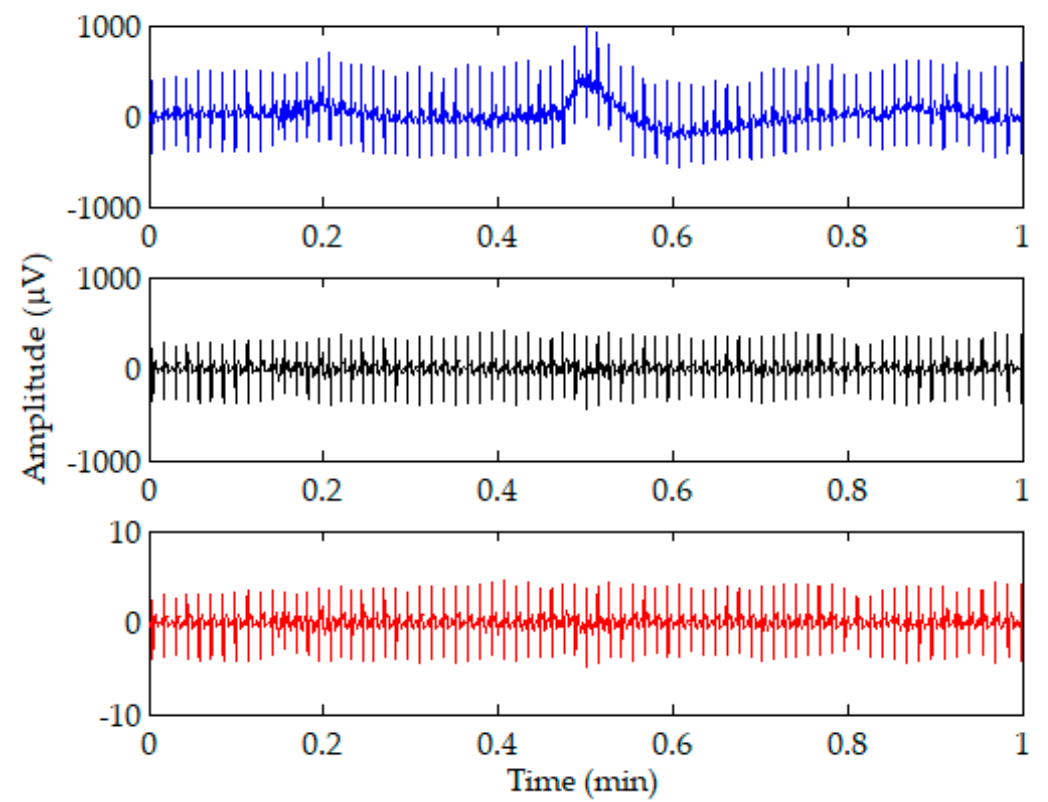

Figure 2. Illustration of a 1-min ECG signal (top) and the signals after bandpass filtering (middle) and z-score normalization (bottom).

The preprocessed 1D ECG signals were then input into the proposed 1D deep CNN model for identifying normal and apnea events. Figure 3 depicts the block diagram of the proposed 1D deep CNN model. It is implemented using TensorFlow [21] and Keras [22]. TensorFlow is a machine learning framework, and also an open source software library that can support various algorithms for deep learning. Keras is a deep learning framework, and also a high-level application programming interface (API) which is capable of running on top of TensorFlow for building, training, and validating deep learning models of neural networks.

The proposed 1D deep CNN model consisted of feature extraction and classification stages. In the feature extraction stage, 10 identical feature extraction layers are designed to extract features from each 1-min ECG signal. Each feature extraction layer includes a 1D CNN layer (Conv-45) with 45 feature maps and a kernel length of 32, a batch normalization layer, an activation layer with the ReLU function, a max pooling layer with a pooling length of 2 , and a dropout layer with a dropout rate of 0.5 . After 10 
feature extraction layers, a flattened layer is used to convert the 2D feature matrix consisting of $451 \mathrm{D}$ feature maps into a $1 \mathrm{D}$ feature vector, to be used by the classifier.

Preprocessed 1-minute ECG

in released and withheld datasets

Feature

Extraction

Layer 1

Feature

Extraction

Layer 10

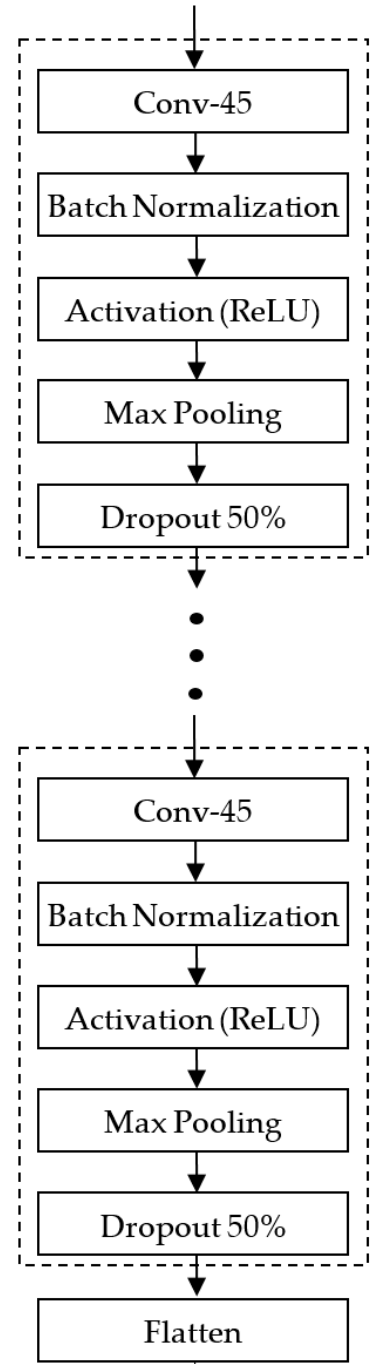

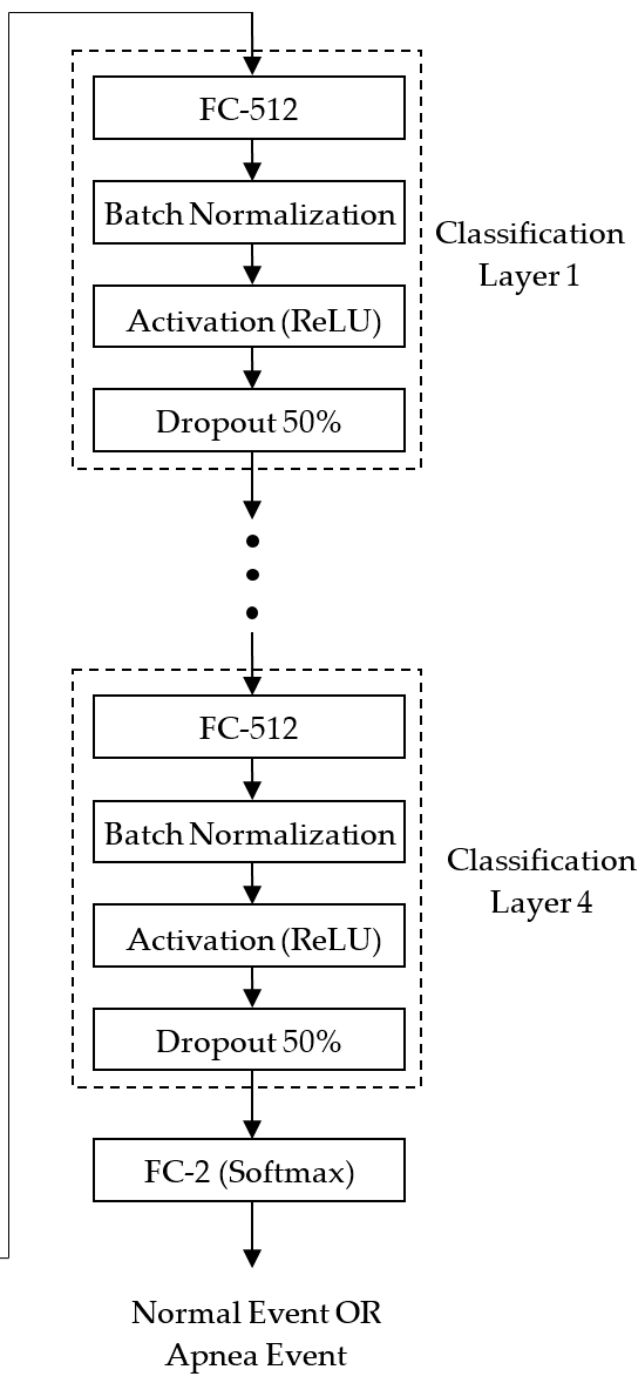

Figure 3. Block diagram of the proposed 1D deep CNN model for identifying normal and apnea events.

In the classification stages, 4 identical classification layers are designed to classify normal and apnea events based on the 1D feature vector. Each classification layer includes a fully connected (FC) layer with 512 neurons, a batch normalization layer, an activation layer with the ReLU function, and a dropout layer with a dropout rate of 0.5 . After 4 classification layers, a softmax activation function is applied to calculate the probabilities of the two outputs of the FC-2 layer. The two outputs correspond to the normal and apnea events, respectively. The result of classification is the group corresponding to the output with a greater probability.

Both the CNN and FC layers use the He normal initialization method [23] to initialize the weights. The weights are initialized taking into account the size of the previous layer of neurons which helps to make the cost function reach the global minimum faster and more efficiently. The batch normalization layers added after the CNN and FC layers both in the feature extraction and classification layers are to 
normalize the data before entering the ReLU activation layer for improving the speed, performance, and stability of the neural network. The max pooling layers in the feature extraction layers are used to reduce the complexity of the network and the possibility of overfitting by selecting the maximum activation in the neighborhood of a neuron in a feature map. The use of the pooling size of 2 reduces the size of each feature map by a factor of 2, e.g., reducing the number of values in each feature map to one half the size. The dropout layers with a dropout rate of 0.5 are used to reduce overfitting by randomly omitting $50 \%$ of the nodes during the training process of the proposed CNN model. The overfitting would cause high training accuracy but low test accuracy. The proposed 1D deep CNN model was trained to minimize cross entropy using the Adam optimizer which is an extension of stochastic gradient descent, and computes individual adaptive learning rates for different parameters from estimates of the first and second moments of the gradients [24].

Table 1 shows the summary of the proposed CNN model. The input layer with the shape of (None, 6000, 1) is used to input a 1-min ECG signal. In each feature-extraction layer, the Conv-45 layer with 45 feature maps adopts a padding parameter of "same," so that each feature map has the same size as the input. Hence the output shape of the Conv-45 layer in the feature extraction layer 1 is (None, 6000, 45). The batch normalization and activation layers do not change the shape of the input. The max pooling layer with a pooling length of 2 and strides of 2 reduces the size of each feature map by half. Hence the output shape of the max pooling layer is reduced to (None, 3000, 45). Although the dropout layer ignores $50 \%$ of the nodes, it does not change the shape of the input. Because each feature extraction layer halves the size of each feature map, the output shape after 10 identical feature extraction layers is reduced to (None, 5, 45). After the flattened layer, a 1D feature vector with 225 features is extracted to be used for the classifier. The output shape of the FC-512 layer with 512 neurons is (None, 512). None of the batch normalization, activation or dropout layers change the shape of the input; hence, after 4 classification layers, the output shape is still (None, 512). The final FC-2 layer with softmax function reduces the output shape to (None, 2).

\section{Results}

\subsection{Performance of the Proposed Apnea Detection System for Per-Minute and Per-Recording Analysis}

The MIT PhysioNet Apnea-ECG containing 70 ECG recordings was used to evaluate the performance of the proposed apnea detection system in order to compare with some previous specific studies which used the same database. The training/released dataset is completely independent of the validation/withheld dataset. The commonly used local performance parameters for the apnea detection system are defined as follows [25]:

$$
\begin{gathered}
\text { Accuracy }=\frac{\mathrm{TP}+\mathrm{TN}}{\mathrm{TP}+\mathrm{FP}+\mathrm{TN}+\mathrm{FN}} \times 100 \% \\
\text { Sensitivity }(\%)=\frac{\mathrm{TP}}{\mathrm{TP}+\mathrm{FN}} \times 100 \% \\
\text { Specificity }(\%)=\frac{\mathrm{TN}}{\mathrm{TN}+\mathrm{FP}} \times 100 \%
\end{gathered}
$$

where TP is the number of true positive events (apnea events predicted as apnea events), FP is the number of false positive events (normal events predicted as apnea events), TN is the number of true negative events (normal events predicted as normal events), and FN is the number of false negative events (apnea events predicted as normal events). This study further plotted the receiver operating characteristic (ROC) to show the apnea detection performance at different classification thresholds, and calculated the area under the ROC curve (AUC) to measure the global performance [26,27]. 
Table 1. Summary of the proposed 1D deep CNN model.

\begin{tabular}{|c|c|c|}
\hline Layers & Parameters & Output Shape \\
\hline Input & & (None, 6000,1 ) \\
\hline \multicolumn{3}{|l|}{ Feature Extraction Layer 1} \\
\hline Conv-45 & $\begin{array}{l}\text { filters }=45, \text { kernel size }=32 \\
\text { padding = 'same' } \\
\text { kernel initializer }=\text { 'he_normal' }\end{array}$ & (None, 6000,45 ) \\
\hline Batch Normalization & & (None, 6000,45 ) \\
\hline Activation & ReLu & (None, 6000,45$)$ \\
\hline Max Pooling & pool size $=2$, strides $=2$ & (None, 3000,45$)$ \\
\hline Dropout & dropout rate $=0.5$ & (None, 3000,45$)$ \\
\hline \multicolumn{3}{|l|}{$\bullet \bullet \bullet$} \\
\hline \multicolumn{3}{|l|}{ Feature Extraction Layer 10} \\
\hline Conv-45 & $\begin{array}{l}\text { filters }=45, \text { kernel size }=32 \\
\text { padding }=\text { 'same' } \\
\text { kernel initializer }=\text { 'he_normal' }\end{array}$ & (None, 11,45 ) \\
\hline Batch Normalization & & (None, 11,45 ) \\
\hline Activation & ReLu & (None, 11,45 ) \\
\hline Max Pooling & pool size $=2$, strides $=2$ & (None, 5,45 ) \\
\hline Dropout & dropout rate $=0.5$ & (None, 5,45 ) \\
\hline Flatten & & (None, 225) \\
\hline \multicolumn{3}{|l|}{ Classification Layer 1} \\
\hline FC-512 & $\begin{array}{l}\text { units }=512 \\
\text { kernel initializer }=\text { 'he_normal' }\end{array}$ & (None, 512) \\
\hline Batch Normalization & & (None, 512) \\
\hline Activation & ReLu & (None, 512) \\
\hline Dropout & dropout rate $=0.5$ & (None, 512) \\
\hline \multicolumn{3}{|l|}{$\bullet \bullet \bullet$} \\
\hline \multicolumn{3}{|l|}{ Classification Layer 4} \\
\hline FC-512 & $\begin{array}{l}\text { units }=512 \\
\text { kernel initializer }=\text { 'he_normal' }\end{array}$ & (None, 512) \\
\hline Batch Normalization & & (None, 512) \\
\hline Activation & ReLu & (None, 512 ) \\
\hline Dropout & dropout rate $=0.5$ & (None, 512) \\
\hline FC-2 & Softmax & (None, 2) \\
\hline
\end{tabular}

Because of the randomness from the weight initialization in the CNN and FC layers and the dropping out in the dropout layer, we repeated 10 training and validation experiments to evaluate the performance of the proposed apnea detection system. The proposed 1D deep CNN model was trained and validated for 50 epochs in each experiment using 1-min ECG signals in the released and withheld datasets, respectively. The batch size was 10. The proposed model included about 1.5 million parameters and required about 402.4 million Multiply-Addition operations, which is about 804.8 million FLOPs. The training and validation of the proposed model were performed on a desktop computer equipped with Windows 10 Professional, Intel(R) Core(TM) i7-9700 3 GHz CPU, 32 GB RAM, and a GeForce(R) RTX2080 Super(TM) 8G graphics card. The training and validation took $61 \mathrm{~s}$ for the first epoch including the weight initialization, and $57 \mathrm{~s}$ for the second to fifth epochs. The time required to complete an experiment was about $48 \mathrm{~min}$. Only $0.3 \mathrm{~ms}$ was required for testing a 1-min ECG signal as a normal or an apnea event using the trained model.

Figure 4 plots the training (blue lines) and validation (orange lines) history curves of the per-minute apnea detection of the proposed 1D deep CNN model for 10 experiments. Figure $4 \mathrm{a}, \mathrm{b}$ are the accuracy 
and loss curves, respectively. The training accuracy and loss for an epoch are the average of the accuracies and losses over each batch of training data, respectively. Hence, we can observe a high degree of consistency from the training history curves of 10 experiments in both the accuracy and loss. However, the validation accuracy and loss for an epoch is computed using the entire validation data and the model, as it is the end of the epoch. The validation history curves of 10 experiments have a relatively larger degree of variation.

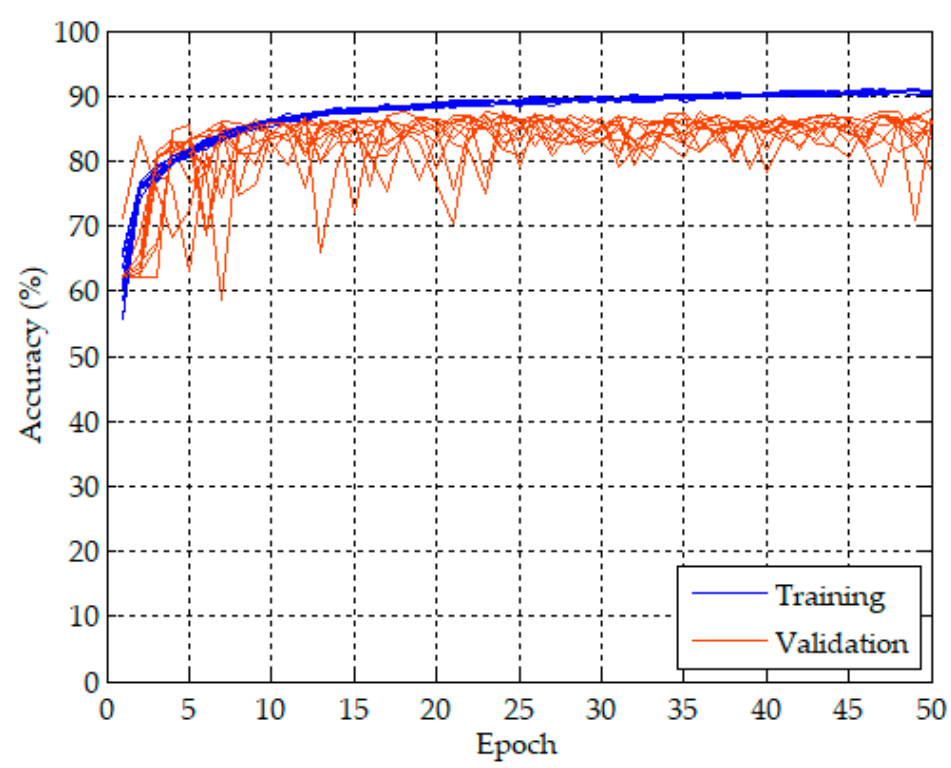

(a)

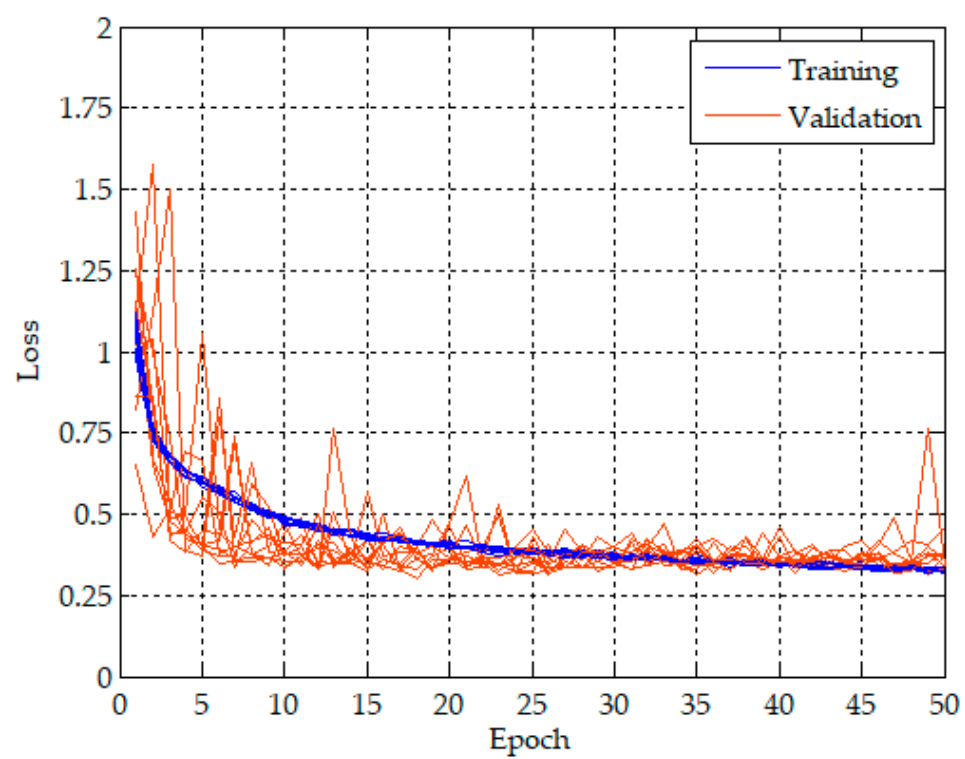

(b)

Figure 4. Training and validation history curves of per-minute apnea analysis for the proposed 1D deep CNN model: (a) accuracy curves, and (b) loss curves.

Figure 5 further shows the best accuracy values of validation in each of the 10 experiments with the corresponding sensitivity, specificity and AUC. The best accuracy values were highly consistent and only ranged from $86.5 \%$ to $87.9 \%$. The corresponding sensitivity, specificity, and AUC ranged from $78.9 \%$ to $87.4 \%$, from $86.8 \%$ to $92.4 \%$, and from $93.1 \%$ to $94.1 \%$, respectively. The best validation accuracy of per-minute apnea detection among the 10 experiments was $87.9 \%$ of the seventh experiment 
with the corresponding sensitivity of $81.1 \%$, specificity of $92.0 \%$, and AUC of $93.5 \%$. Figure 6 plots the ROC curves corresponding to the model with the best validation accuracy values of per-minute apnea detection in each of the 10 experiments. The 10 ROC curves showed a high degree of consistency of validation. Hence, except that the training results are highly reproducible in Figure 4, the best validation accuracy values in Figure 5 and the corresponding ROC curves in Figure 6 also demonstrate the high reproducibility of the validation results in the 10 experiments. Table 2 shows a summary of the confusion matrix and performance parameters of per-minute apnea detection for the training/released and validation/withheld datasets. The accuracy can reach $93.4 \%$ with sensitivity of $91.5 \%$ and specificity of $94.6 \%$ for the entire training/released dataset using the model with the best validation accuracy of $87.9 \%$.

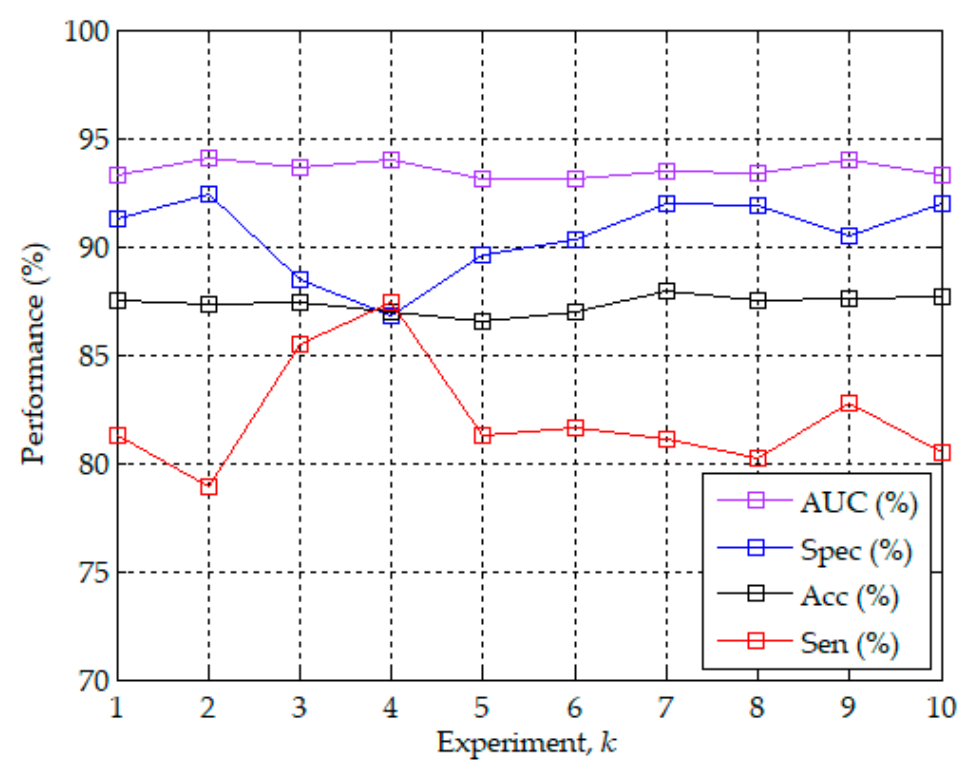

Figure 5. The best validation accuracy values of per-minute apnea analysis in each of the 10 experiments with the corresponding sensitivity, specificity, and the area under the (receiver operating characteristic (ROC)) curve (AUC).

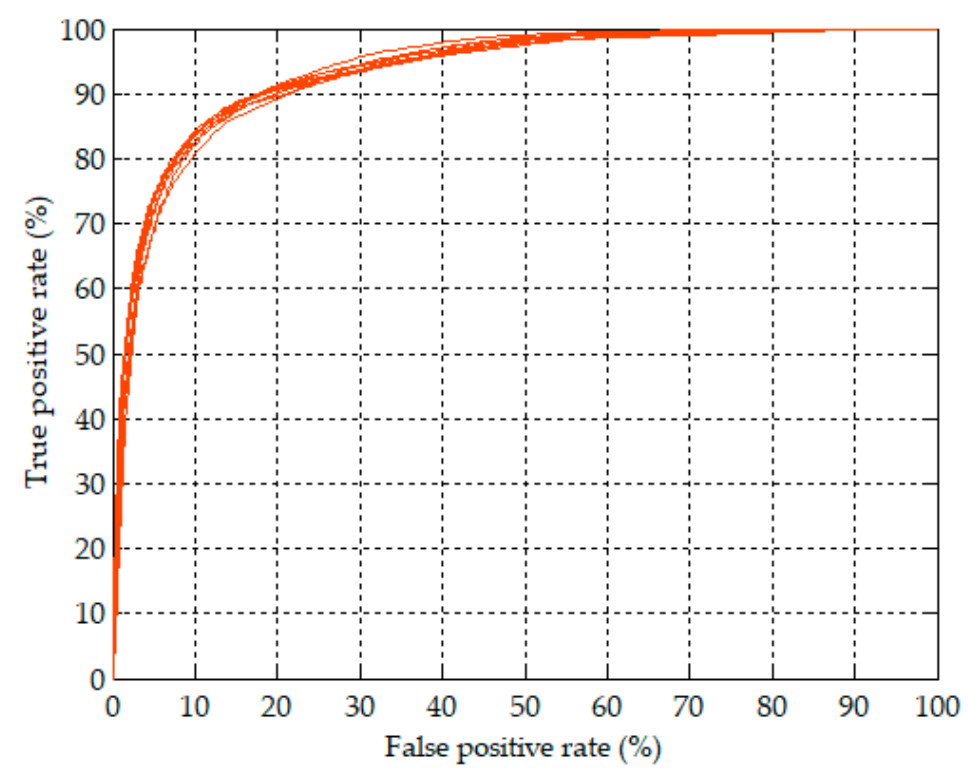

Figure 6. ROC curves corresponding to the model with the best validation accuracy values of per-minute apnea analysis in each of the 10 experiments. 
Table 2. Summary of the confusion matrix and performance parameters of per-minute apnea analysis for the training/released and validation/withheld datasets.

\begin{tabular}{|c|c|c|c|c|c|c|}
\hline Dataset & Label Predict & $\mathbf{N}$ & A & Sen $(\%)$ & Spe (\%) & $\operatorname{Acc}(\%)$ \\
\hline \multirow[t]{2}{*}{ Training/Released } & $\mathrm{N}$ & 9760 & 562 & 91.5 & 94.6 & 93.4 \\
\hline & A & 565 & 6092 & & & \\
\hline \multirow[t]{2}{*}{ Validation/Withheld } & $\mathrm{N}$ & 9863 & 854 & 81.1 & 92.0 & 87.9 \\
\hline & $\mathrm{A}$ & 1230 & 5287 & & & \\
\hline
\end{tabular}

N: Normal event; A: Apnea event; Sen: Sensitivity; Spe: Specificity; and Acc: Accuracy.

Based on the results of the per-minute apnea detection, we can further diagnose an ECG recording as a non-OSA subject or an OSA patient. According to the recommendation of the American Academy of Sleep Medicine (AASM), the OSA syndrome is defined as an AHI of five or greater [28]. The AHI value of each ECG recording is estimated by the results of per-minute apnea detection, defined as follows:

$$
\mathrm{AHI}=\frac{60}{L} \times N
$$

where $L$ denotes the number of 1-min ECG signals for a recording, $L / 60$ is the number of hours for a recording, and $N$ is the number of 1-min signals which are detected as apnea events.

Table 3 summarizes the results of the per-recording analysis for the training/released and validation/withheld datasets. Both datasets have 35 ECG recordings. The recordings with AHI greater than or equal to five were diagnosed as OSA. The two datasets had the same diagnostic performance of per-recording analysis with accuracy of $97.1 \%$, sensitivity of $95.7 \%$, and specificity of $100 \%$. Only one OSA patient was misdiagnosed as a non-OSA subject in both datasets. The correlation coefficients between the estimated AHI values of the proposed CNN model and the actual PSG AHI values provided by the MIT PhysioNet apnea-ECG database were 0.938 and 0.865 for the training/released and validation/withheld datasets, respectively.

Table 3. Summary of the results of the per-recording analysis for the training/released and validation/withheld datasets.

\begin{tabular}{ccccccc}
\hline Dataset & Recordings & Diagnostic Criteria & Sen (\%) & Spe (\%) & Acc (\%) & Corr. \\
\hline Training/Released & 35 & 5 & 95.7 & 100 & 97.1 & 0.938 \\
\hline Validation/Withheld & 35 & 5 & 95.7 & 100 & 97.1 & 0.865 \\
\hline
\end{tabular}

Sen: Sensitivity; Spe: Specificity; Acc: Accuracy; and Corr.: Correlation.

\subsection{The Effect of the Number of Feature Extraction Layers on the Performance of Apnea Detection}

In addition to the apnea detection performance of the proposed CNN model using 10 feature extraction layers demonstrated above, we further analyzed the effect of the number of feature extraction layers on the performance. We evaluated the performance of the proposed CNN model using 1 to 12 feature extraction layers, respectively. Because each feature extraction layer included a max pooling layer with a pooling length of two, the more feature extraction layers, the fewer the extracted features. These CNN models used the same four FC-based classification layers. Each CNN model repeated 10 experiments. Figure 7 plots the curves of the number of feature extraction layers vs. The best accuracy in 10 experiments with the corresponding specificity, sensitivity, and AUC. It can be observed that the model using only six feature extraction layers achieved the accuracy of $87.4 \%$ with the corresponding specificity of $91.1 \%$, sensitivity of $81.3 \%$, and AUC of $92.8 \%$. The accuracy and the corresponding AUC slightly increased with the number of feature extraction layers from 6 to 10 layers, and slightly decreased after 10 layers. The specificity and sensitivity did not increase with the layers. A higher specificity was accompanied by a lower sensitivity, and vice versa. The proposed 
CNN model with 10 feature extraction layers had the best accuracy of $87.9 \%$ among the models using 1 to 12 feature extraction layers.

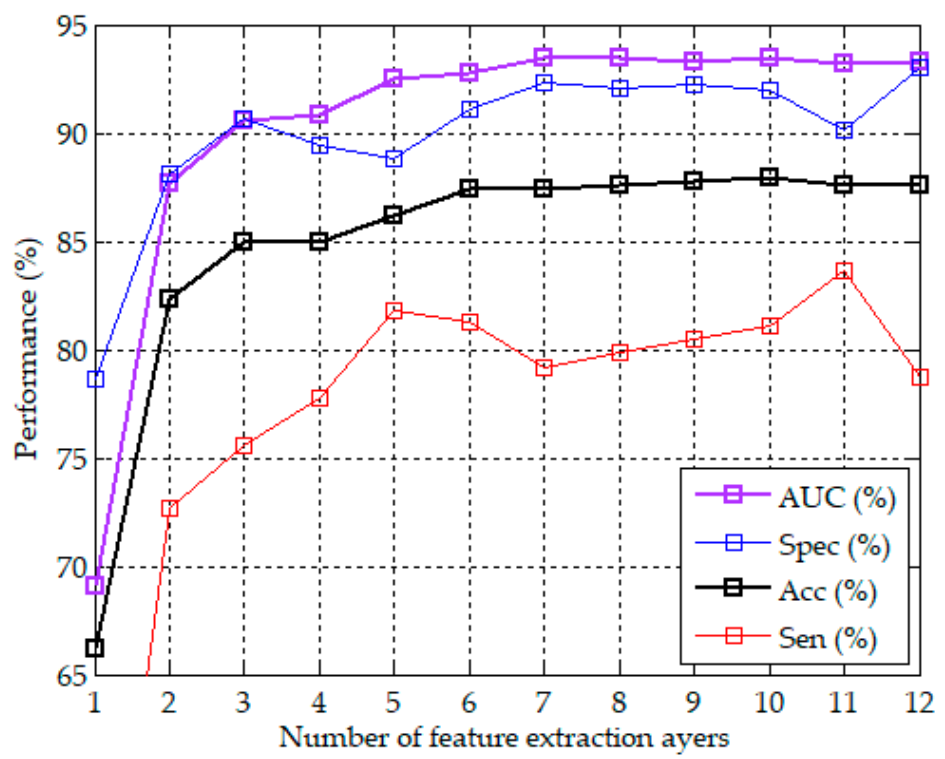

Figure 7. Curves of the number of feature extraction layers vs. The best accuracy in 10 experiments with the corresponding specificity, sensitivity, and AUC.

\section{Discussion}

The study has demonstrated that the proposed apnea detection system based on a 1D deep CNN model can extract the features from 1-min ECG signals and classify them into normal and apnea events. The released and withheld datasets each with 35 ECG recordings from the MIT PhysioNet apnea-ECG database were used to train and validate the proposed system. The input signals are the 1D ECG signals. The signal preprocessing only needs the Butterworth band-pass filtering and z-score normalization. The proposed 1D deep CNN model includes 10 identical feature extraction layers, a flattened layer, 4 identical classification layers, and a softmax FC layer. A total of 10 1D-CNN layers were designed to extract the features of the 1-min ECG signal, and a total of 5 FC layers were designed to classify the normal and apnea events based on the extracted features. The feature extractor and classifier are trained together by the Adam optimizer [24].

Several studies have proposed apnea detection approaches based on the features extracted from the RR intervals or heart rates [10-14]. However, the calculation of the RR intervals or heart rates requires an accurate QRS detection algorithm. A wrong $R$ peak would cause one or two wrong RR intervals which are difficult to correct, thus reducing the accuracy of the extracted features. In contrast, this study only used the original 1D ECG signals as the input, without the inclusion of RR intervals or heart rates. Neither the detection of the QRS complexes nor the analysis of RR intervals or heart rates were required in this study. Moreover, most of the previous work was focused on feature engineering which needs prior knowledge of OSA to extract features using a particular method [7-14]. This means that the feature engineering process needs a large amount of labor to determine the most representative features of apnea events. In this study, the feature extraction and classifier are designed and trained together, and the features can be automatically extracted by the proposed CNN model.

Table 4 compares the signal preprocessing methods of the proposed apnea system with three feature-learning-based and three feature-engineering-based systems. These studies all adopted the MIT PhysioNet apnea-ECG database, and the released and withheld datasets were used for training and validation, respectively. Our study and the studies of Singh and Majumder [15], Wang et al. [16], and $\mathrm{Li}$ et al. [17] proposed feature-learning-based methods which can automatically learn the features of ECG signals or RR intervals using neural networks. The studies of Sharma and Sharma [12], 
Song et al. [13], and Varon et al. [14] focused on feature-engineering-based methods. Only our study and the study of Singh and Majumder [15] did not require R-peaks detection and RR interval calculation. However, the apnea detection system proposed by Singh and Majumder [15] is based on a 2D CNN model, and thus a continuous wavelet transform is required to transfer the 1D ECG signals to 2D scalogram images in the signal preprocessing stage. In comparison with the 1D CNN model, the 2D $\mathrm{CNN}$ model needs more parameters and has higher computational costs. Because the proposed CNN model has the same dimension of 1D as the input ECG signals, the signal preprocessing does not require additional signal transformation in this study. The other studies all required R-peaks detection and RR interval calculation. The media filtering $[13,16,17]$ and R-peaks correction [14] have often been used to eliminate the physiologically uninterpretable points. Interpolation $[16,17]$ has been used to interpolate all RR interval series to have the same length. The QRS complex extraction and EDR derivation were also included in the studies of Sharma and Sharma [12], and Song et al. [13], and Varon et al. [14], respectively. Accordingly, in comparison with these studies [12-17], the proposed apnea detection system can simplify the complexity of the signal preprocessing, only including bandpass filtering and z-score normalization.

Table 4. Comparison of the signal preprocessing methods of the proposed apnea system with the previous studies.

\begin{tabular}{cl}
\hline Reference & \multicolumn{1}{c}{ Signal Preprocessing Methods } \\
\hline Our Study & \multicolumn{1}{c}{ Feature-Learning-Based Systems } \\
\hline Singh and Majumder [15] & $\begin{array}{l}\text { Bandpass Filtering + Z-score Normalization } \\
\text { Zerocenter Normalization }\end{array}$ \\
\hline \multirow{2}{*}{ Wang et al. [16] } & FIR Filtering + R-peaks Detection + \\
& RR Interval Calculation + Median Filtering + \\
& Cubic Interpolation \\
\hline \multirow{2}{*}{ Li et al. [17] } & Bandpass Filtering + R-peaks Detection + \\
& RR Interval Calculation + Median Filtering + \\
& Interpolation \\
\hline \multirow{2}{*}{ Sharma and Sharma [12] } & Feature-Engineering-Based Systems \\
& Bandpass Filtering + R-peaks Detection + \\
& RR Interval Calculation + QRS Complex Extraction + Zero Padding \\
\hline \multirow{2}{*}{ Song et al. [13] } & Filter-Bank-Based R-peaks Detection + \\
& RR Interval Calculation + Median Filtering + \\
& EDR Derivation \\
\hline Varon et al. [14] & Notch Filtering + DC Component Remove + \\
& Up Sampling + R-peaks Detection + \\
& R-peaks Correction + RR Interval Calculation + \\
& EDR Derivation \\
\hline
\end{tabular}

FIR: Finite impulse response; DC: Direct current; and EDR: ECG-derived respiration.

Table 5 compares the performance of the proposed 1D deep CNN model with the previous studies for the per-minute apnea detection. The sensitivity, specificity, accuracy, and AUC of all studies ranged from $79.5 \%$ to $90.0 \%$, from $82.1 \%$ to $92.0 \%$, from $83.8 \%$ to $87.9 \%$, and from 0.83 to 0.95 , respectively, for the per-minute apnea detection. The best accuracy is the $87.9 \%$ of the proposed method, followed by the $87.6 \%$ of Wang et al. [16], and the $86.2 \%$ of Singh and Majumder [15] and Song et al. [13]. The proposed method also has the best specificity of $92.0 \%$, followed by the $90.3 \%$ of Wang et al. [16], and the $88.4 \%$ of Sharma and Sharma [12] and Song et al. [13]. Singh and Majumder [12] has the best sensitivity of $90.0 \%$, but their specificity is only $83.8 \%$. The sensitivity of Li et al. [17] is the second highest at $88.9 \%$, which is only slightly lower than the $90 \%$ of Singh and Majumder [15], but their specificity is also only $82.1 \%$. The best AUC is the 0.95 of Wang et al. [16], which is slightly higher than 
the 0.94 of the proposed method and of Song et al. [13]. The AUC values of other studies are all lower than 0.90 .

Table 5. Performance comparison of the proposed 1D deep CNN model with the previous studies for the per-minute apnea detection.

\begin{tabular}{cccccc}
\hline \multicolumn{1}{c}{ Reference } & Methods & Sen (\%) & Spe (\%) & Acc (\%) & AUC \\
\hline \multicolumn{7}{c}{ Feature-Learning-Based Methods } & & & \\
\hline Our Study & The proposed 1D Deep CNN Model & 81.1 & 92.0 & 87.9 & 0.94 \\
\hline $\begin{array}{c}\text { Singh and } \\
\text { Majumder [15] }\end{array}$ & $\begin{array}{c}\text { Pre-trained AlexNet CNN + } \\
\text { Decision Fusion }\end{array}$ & 90.0 & 83.8 & 86.2 & 0.88 \\
\hline Wang et al. [16] & LeNet-5 CNN & 83.1 & 90.3 & 87.6 & 0.95 \\
\hline Li et al. [17] & Auto-encoder + Decision Fusion & 88.9 & 82.1 & 84.7 & 0.87 \\
\hline \multicolumn{7}{c}{ Sharma and } \\
Sharma [12] & Feature-Engineering-Based Methods & & & \\
\hline Song et al. [13] & Feature Engineering + HMM-SVM & 82.6 & 88.4 & 86.2 & 0.94 \\
\hline Varon et al. [14] & Feature Engineering + LS-SVM & 84.7 & 84.7 & 84.7 & 0.88 \\
\hline
\end{tabular}

Table 6 further compares the performance of the proposed 1D deep CNN model with the previous studies for the per-recording classification. The performance of per-recording classification of the study of Varon et al. [14] is not listed in Table 6. They divided 70 recordings into normal, borderline, and apnea classes. The AHI was computed for each recording. Their results showed that the normal subjects could be separated from apnea patients with an accuracy of $100 \%$ if AHI of 10 or greater was used to diagnose apnea patients. However, they did not provide the details of the borderline subjects. All of the diagnostic criteria shown in Table 6 were the estimated AHI of five or greater. The withheld dataset includes 35 recordings consisting of 23 OSA patients and 12 non-OSA subjects. The best accuracy is the $100 \%$ of Singh and Majumder [15] and Li et al. [17]. In our study, an OSA patient was misclassified as a non-OSA subject. Hence, the accuracy of the proposed approach is $97.1 \%$ with sensitivity of $95.7 \%$ and specificity of $100 \%$. The studies of Sharma and Sharma [12] and Song et al. [13] have the same performance as our study. Their sensitivity of $95.8 \%$ should be corrected to $95.7 \%$. In the study of Wang et al. [16], a non-OSA subject was misclassified as an OSA patient. Hence, their accuracy is $97.1 \%$, with sensitivity of $100 \%$ and specificity of $91.7 \%$.

Table 6. Performance comparison of the proposed apnea detection system with the previous studies for the per-recording classification.

\begin{tabular}{|c|c|c|c|c|c|c|}
\hline Reference & Recordings & $\begin{array}{c}\text { Diagnostic } \\
\text { Criteria }\end{array}$ & Sen $(\%)$ & Spe $(\%)$ & Acc (\%) & Corr. \\
\hline Our study & \multirow{6}{*}{$\begin{array}{c}23 \\
\text { OSA Patients } \\
+ \\
12 \\
\text { Healthy Subjects }\end{array}$} & \multirow{6}{*}{5} & 95.7 & 100 & 97.1 & 0.865 \\
\hline Singh and Majumder [15] & & & 100 & 100 & 100 & - \\
\hline Wang et al. [16] & & & 100 & 91.7 & 97.1 & 0.943 \\
\hline Li et al. [17] & & & 100 & 100 & 100 & - \\
\hline Sharma and Sharma [12] & & & 95.8 & 100 & 97.1 & 0.841 \\
\hline Song et al. [13] & & & 95.8 & 100 & 97.1 & 0.860 \\
\hline
\end{tabular}

The studies of Singh and Majumder [15] and Li et al. [17] did not provide the correlation coefficients between the estimated and actual PSG AHI values. The correlation coefficient of the proposed model is 0.865, which is slightly higher than the 0.860 of Song et al. [13] and the 0.841 of Sharma and Sharma [12]. It is worth noting that Wang et al. [16] had a much higher correlation coefficient of 0.943 , but lower accuracy for the per-minute apnea detection in comparison with our study. This inconsistent result may be caused by the inconsistent definition of the correlation coefficient. If we calculate the correlation 
coefficient between the estimated AHI values and the AHI values according to per-minute annotations, it is 0.948 for the proposed model, which is slightly higher than the 0.943 of Wang et al. [16]. Hence, it is possible that the correlation coefficient in Wang et al. [16] is calculated between the estimated AHI values and the AHI values according to the annotations of the 1-min ECG signals, not between the estimated AHI values and the actual PSG AHI values.

The limitation of the study is the lower sensitivity of $81.1 \%$ for per-minute apnea detection in comparison with the other studies listed in Table 5. A lower sensitivity would cause more apnea events to be misidentified as normal events, and hence would lower the estimated AHI and may cause OSA patients to be misclassified as non-OSA subjects for the per-recording classification. Accordingly, our future work will focus on enhancing the sensitivity of the per-minute apnea detection for further increasing the feasibility of using a single-lead ECG to detect apnea events and diagnose OSA patients.

\section{Conclusions}

This study proposes a 1D deep CNN model for the detection of apnea events only using 1D ECG signals as input. The proposed CNN model includes $10 \mathrm{CNN}$-based feature extraction layers and 4 FC-based classification layers. The signal preprocessing only needs Butterworth bandpass filtering and z-score normalization without the detection of QRS complexes, the analysis of RR intervals, or additional signal transformation. The proposed CNN model was trained and validated by the released and withheld datasets of the MIT PhysioNet Apnea-ECG database, respectively. In comparison with several previous studies, the proposed method has the best accuracy of $87.9 \%$ and specificity of $92.0 \%$ with corresponding sensitivity of $81.1 \%$ and AUC of 0.94 for per-minute apnea detection. The performance of per-recording classification can achieve the accuracy of $97.1 \%$ with sensitivity of $95.7 \%$ and specificity of $100 \%$. The proposed system can be served as a convenient and advanced diagnosis system of OSA only using 1D ECG signals. If the estimated AHI is greater than or equal to five, it is recommended to follow up with a PSG test to confirm the severity of OSA.

Author Contributions: Conceptualization, H.-Y.C. and C.-C.L.; methodology, C.-Y.Y. and C.-C.L.; software, C.-T.L. and C.-C.L.; validation, H.-Y.C., C.-Y.Y., C.-T.L., and C.-C.L.; formal analysis, C.-Y.Y.; investigation, H.-Y.C.; resources, C.-T.L.; data curation, C.-T.L.; writing-original draft preparation, H.-Y.C. and C.-C.L.; writing-review and editing, C.-C.L.; visualization, C.-Y.Y.; supervision, H.-Y.C.; project administration, C.-Y.Y.; and funding acquisition, C.-C.L. All authors have read and agreed to the published version of the manuscript.

Funding: This research was funded by Ministry of Science and Technology of the Republic of China, Taiwan, grant number MOST 108-2637-E-167-002-.

Conflicts of Interest: The authors declare no conflict of interest.

\section{References}

1. Mannarino, M.R.; Filippo, F.D.; Pirro, M. Obstructive sleep apnea syndrome. Eur. J. Intern. Med. 2012, 23, 586-593. [CrossRef]

2. Javaheri, S.; Barbe, F.; Campos-Rodriguez, F.; Dempsey, J.A.; Khayat, R.; Javaheri, S.; Malhotra, A.; Martinez-Garcia, M.A.; Mehra, R.; Pack, A.I.; et al. Sleep apnea: Types, mechanisms, and clinical cardiovascular consequences. J. Am. Coll. Cardiol. 2017, 69, 841-858. [CrossRef] [PubMed]

3. Chesson, A.L.; Ferber, R.A.; Fry, J.M.; Grigg-Damberger, M.; Hartse, K.M.; Hurwitz, T.D.; Johnson, S.; Littner, M.; Kader, G.A.; Rosen, G.; et al. Practice parameters for the indications for polysomnography and related procedures. Sleep 1997, 20, 406-422. [CrossRef] [PubMed]

4. Kakkar, R.K.; Berry, R.B. Positive airway pressure treatment for obstructive sleep apnea. Chest 2007, 132, 1057-1072. [CrossRef] [PubMed]

5. Hudgel, D.W. Sleep apnea severity classification-revisited. Sleep 2016, 39, 1165-1166. [CrossRef]

6. Mendonc, F.; Mostafa, S.S.; Ravelo-García, A.G.; Morgado-Dias, F.; Penzel, T. A review of obstructive sleep apnea detection approaches. IEEE J. Biomed. Health. Inform. 2019, 23, 825-837. [CrossRef]

7. Lin, R.; Lee, R.; Tseng, C.; Zhou, H.; Chao, C.; Jiang, J. A new approach for identifying sleep apnea syndrome using wavelet transform and neural networks. Biomed. Eng. Appl. Basis Commun. 2006, 18, 138-143. [CrossRef] 
8. Rachim, V.; Li, G.; Chung, W. Sleep apnea classification using ECG signal wavelet-PCA features. Bio-Med. Mater. Eng. 2014, 24, 2875-2882. [CrossRef]

9. Hassan, A.; Haque, A. An expert system for automated identification of obstructive sleep apnea from single-lead ECG using random under sampling boosting. Neurocomputing 2017, 235, 122-130. [CrossRef]

10. Nguyen, H.D.; Wilkins, B.A.; Cheng, Q.; Benjamin, B.A. An online sleep apnea detection method based on recurrence quantification analysis. IEEE J. Biomed. Health Inform. 2014, 18, 1285-1293. [CrossRef]

11. Atri, R.; Mohebbi, M. Obstructive sleep apnea detection using spectrum and bispectrum analysis of single-lead ecg signal. Physiol. Meas. 2015, 36, 1963-1980. [CrossRef] [PubMed]

12. Sharma, H.; Sharma, K.K. An algorithm for sleep apnea detection from single-lead ECG using hermite basis functions. Comput. Biol. Med. 2016, 77, 116-124. [CrossRef]

13. Song, C.; Liu, K.; Zhang, X.; Chen, L.; Xian, X. An obstructive sleep apnea detection approach using a discriminative hidden markov model from ECG signals. IEEE Trans. Biomed. Eng. 2016, 63, 1532-1542. [CrossRef] [PubMed]

14. Varon, C.; Caicedo, A.; Testelmans, D.; Buyse, B.; Huffel, S.V. A novel algorithm for the automatic detection of sleep apnea from single-lead ECG. IEEE Trans. Biomed. Eng. 2015, 62, 2269-2278. [CrossRef] [PubMed]

15. Singh, S.A.; Majumder, S. A novel approach OSA detection using single-lead ECG scalogram based on deep learning network. J. Mech. Med. Biol. 2019, 19, 1-18. [CrossRef]

16. Wang, T.; Lu, C.; Shen, G.; Hong, F. Sleep apnea detection from a single-lead ECG signal with automatic feature-extraction through a modified LeNet-5 convolutional neural network. PeerJ 2019, 7, e7731. [CrossRef] [PubMed]

17. Li, K.; Pan, W.; Li, Y.; Jiang, Q.; Liu, G. A method to detect sleep apnea based on deep neural network and hidden Markov model using single-lead ECG signal. Neurocomputing 2018, 294, 94-101. [CrossRef]

18. Penzel, T.; Moody, G.B.; Mark, R.G.; Goldberger, A.L.; Peter, J.H. The apnea-ECG database. Comput. Cardiol. 2000, 27, 255-258.

19. Goldberger, A.L.; Amaral, L.A.N.; Glass, L.; Hausdorff, J.M.; Ivanov, P.C.; Mark, R.G.; Mietus, J.E.; Moody, G.B.; Peng, C.-K.; Stanley, H.E. PhysioBank, PhysioToolkit, and PhysioNet: Components of a new research resource for complex physiologic signals. Circulation 2003, 101, e215-e220. [CrossRef]

20. Signal Processing Toolbox-MATLAB. Available online: https://www.mathworks.com/products/signal.html (accessed on 19 June 2019).

21. Abadi, M.; Agarwal, A.; Barham, P.; Brevdo, E.; Chen, Z.; Citro, C.; Corrado, G.; Davis, A.; Dean, J.; Devin, M.; et al. TensorFlow: Large-scale machine learning on heterogeneous distributed systems. arXiv 2016, arXiv:1603.04467. Available online: https://arxiv.org/abs/1603.04467 (accessed on 25 July 2020).

22. Keras: Deep Learning for Humans. Available online: https://github.com/fchollet/keras (accessed on 19 June 2019).

23. He, K.; Zhang, X.; Ren, S.; Sun, J. Delving deep into rectifiers: Surpassing human-level performance on ImageNet classification. arXiv 2015, arXiv:1502.01852. Available online: https://arxiv.org/abs/1502.01852 (accessed on 25 July 2020).

24. Kingma, P.D.; Ba, J.L. Adam: A method for stochastic optimization. arXiv 2017, arXiv:1412.6980v9. Available online: https://arxiv.org/abs/1412.6980v9 (accessed on 25 July 2020).

25. Griner, P.F.; Mayewski, R.J.; Mushlin, A.I.; Greenland, P. Selection and interpretation of diagnostic tests and procedures. Principles and applications. Ann. Intern. Med. 1981, 94, 557-592.

26. Metz, C.E. Basic principles of ROC analysis. Semin. Nucl. Med. 1978, 8, 283-298. [CrossRef]

27. Zweig, M.H.; Campbell, G. Receiver-operating characteristic (ROC) plots: A fundamental evaluation tool in clinical medicine. Clin. Chem. 1993, 39, 561-577. [CrossRef] [PubMed]

28. American Academy of Sleep Medicine. European Respiratory Society; Australasian Sleep Association; American Thoracic Society; Sleep-related breathing disorders in adults: Recommendations for syndrome definition and measurement techniques in clinical research: The report of an American Academy of Sleep Medicine Task Force. Sleep 1999, 22, 667-689.

(C) 2020 by the authors. Licensee MDPI, Basel, Switzerland. This article is an open access article distributed under the terms and conditions of the Creative Commons Attribution (CC BY) license (http://creativecommons.org/licenses/by/4.0/). 